\title{
Selected Laser-based Therapies in Otolaryngology
}

Silvia Mioc, $\mathrm{PhD}, \mathrm{MBA}^{\mathrm{a}, \mathrm{b}, *}$, Mary-Ann Mycek, $\mathrm{PhD}^{\mathrm{c}, \mathrm{d}}$

${ }^{\text {a }}$ Colorado Photonics Industry Association, 105 South Sunset Street, Suite G, Longmont, CO 80501, USA

${ }^{\mathrm{b}}$ General Electric Healthcare Technologies, Louisville, CO, USA

${ }^{\mathrm{c}}$ Applied Physics Program, Department of Biomedical Engineering, College of Engineering, University of Michigan, 2200 Bonisteel Boulevard, Ann Arbor, MI 48109, USA

${ }^{\mathrm{d}}$ Comprehensive Cancer Center, University of Michigan, Ann Arbor, MI, USA

Lasers have found a natural place in medicine for both therapeutic and diagnostic applications. Their use depends on the unique characteristics of the laser light and the on the interaction of the laser with tissue. In general, light incident on tissue can be absorbed, reflected, multiply scattered, or transmitted. Optical diagnostic modalities frequently use reflected and scattered light, whereas the energy from light needs to be absorbed to induce a clinical effect and achieve therapy. Reinisch [1] has written a comprehensive review of the fundamentals of laser properties and their interaction with biologic tissues.

This article provides an overview of selected laser-based therapies used in otolaryngology. The selection complements a recent review [2].

Because otolaryngologists are increasingly treating vascular lesions of the face, the first section of this article addresses the treatment of vascular lesions of the face with focus on the lesion most commonly treated using lasers, the port-wine stain (PWS). The discussion includes an introduction of laser-tissue interactions and recent clinical and modeling advances in the field.

The second section discusses the use of photodynamic therapy (PDT) in otolaryngology. The discussion of PDT includes an introduction to the basic theory of photodynamic action, including special considerations for in vivo tissue therapeutics; an illustration of the approach using representative clinical applications of PDT in otolaryngology, including recent advances in

* Corresponding author. Colorado Photonics Industry Association, 105 South Sunset Street, Suite G, Longmont, CO 80501, USA.

E-mail address: Silvia.Mioc@comcast.net (S. Mioc). 
the field; and a discussion of the cost-effectiveness of PDT relative to traditional therapies.

\section{Recent advances in treatment of vascular lesions of the face with focus on port-wine stains}

PWSs are benign vascular birthmarks consisting of superficial and deep dilated capillaries in the skin resulting in a reddish to purplish discoloration. They are present in $0.3 \%$ to $0.5 \%$ of newborns, initially are light in color, darken with age as capillaries continue to dilate, and can later progress to a raised and nodular surface. PWSs can cause significant psychologic trauma and often lead to a reduction in the quality of life. Treatment for PWS has included skin grafting, ionizing radiation, cryosurgery, tattooing, dermabrasion, and laser treatments [3]. Currently, lasers provide the treatment of choice for most PWS patients.

\section{Interaction of laser light with skin tissue}

The interaction of light with tissue is affected by two processes: absorption and scattering. Absorption is dominant in the epidermis, and scattering by collagen fibers is dominant in the dermis.

Anything that has color will preferentially absorb some region of visible light, and many objects that appear transparent absorb infrared light. For skin tissue, the main chromophores are proteins and DNA for shortwavelength UV light, melanin and hemoglobin for visible light, and water, the main component of tissue, for long-wavelength infrared light. The depth of light penetration is usually defined as the length over which the incident energy drops by $1 / \mathrm{e}$, or about $64 \%$. Over the visible region of the spectrum, light penetration is inversely related to wavelength. On average, visible light penetrates tissue to a depth of $1 \mathrm{~mm}$; light with a wavelength around 1 micron $(\mathrm{Nd}$ :YAG laser, $1.06 \mu \mathrm{m})$ penetrates most deeply, to a depth of about $4 \mathrm{~mm}$; as the wavelength increases, the depth of penetration drops to $30 \mu \mathrm{m}$ for the $\mathrm{CO}_{2}$ laser $(10.6 \mu \mathrm{m})$. (These numbers represent an average depth of penetration for several tissues, not for any particular tissue [1]).

When chromophores absorb light, their molecules become excited, and the absorbed energy can be dissipated as heat, a photothermal effect. A $5^{\circ}$ to $10^{\circ} \mathrm{C}$ increase in temperature leads to cell injury, temperatures close to $100^{\circ} \mathrm{C}$ cause denaturation of DNA and proteins, temperatures above $100^{\circ} \mathrm{C}$ can ablate tissue through the vaporization of intracellular water, and further heating leads to desiccation and charring [4]. The heat deposited in the target chromophore will dissipate to adjacent areas, a process called "thermal relaxation." The rate of cooling depends on the thermal relaxation time, $t$, defined as the time required to cool the target to one half of its peak temperature immediately after laser irradiation. The extent of thermal damage depends on the temperature reached in the target tissue (which 
relates to the laser wavelength and fluence/energy density) and on the length of time that the tissue remains at that temperature (which relates to the duration of the laser pulse and t). The photothermal effect in its ablative manifestation is the main mechanism for the laser scalpel. In its nonablative manifestation, the photothermal effect is the mechanism used to treat vascular lesions, including PWSs, as discussed in more detail later in this article.

Localized heating caused by absorption can cause acoustic waves that mechanically destroy the absorbing tissue, a photomechanical effect. This effect may be the mechanism that leads to vascular rupture and purpura in PWS treatment with very short pulses (1 microsecond) and to pigment destruction in tattoo removal [4].

Last, absorbed laser energy can induce photochemical changes in tissues. This effect is the basis for photodynamic therapy (PDT), as discussed in the last part of this article.

\section{Therapeutic mechanisms of lasers for port-wine stains}

The goal in treating a PWS with a laser is to destroy the underlying blood vessels selectively (thus lightening the birth mark) without affecting the surrounding tissue and causing scarring. For this selective photothermolysis one must find a laser not absorbed by the skin and preferentially absorbed in the blood vessels at a rate faster than their cooling rate [5].

The main absorbers in the blood vessels are oxyhemoglobin and reduced hemoglobin. Oxyhemoglobin absorbs in the visible-light range, with peaks in the blue and yellow region $(577 \mathrm{~nm})$ of the spectrum. Reduced hemoglobin absorbs in the infrared range of the spectrum. Because venous saturation is approximately $70 \%$, oxyhemoglobin is dominant, and visible lasers have generally been used for treatment. The yellow region happens to be a window of low absorption for melanin. Thus, in principle, for lightcolored skin, a yellow laser will be absorbed preferentially by red blood cells, with minimal absorption in the skin. This preferential absorption is advantageous for two reasons. First, minimal direct thermal damage is caused to the epidermis, because only a small part of the energy is deposited there. Second, more light can reach the blood vessels to cause thrombosis and thus lightening of the PWS. To limit the thermal damage to the skin, the exposure of the skin to the laser should be shorter than t; therefore, pulsed lasers with pulse durations not longer than $t$ are the treatment of choice. For dark skin, however, the melanin absorption is so high that thrombosis of the vessels is hard to obtain without damage to the skin. In this case, using infrared lasers, such as the Nd:YAG laser $(1.06 \mu \mathrm{m})$, with light absorbed primarily by the reduced hemoglobin, is preferred.

The size of the vessels determines the amount of energy to be deposited for thrombosis to take place, and thus the combination of the energy density (fluence) and length of the pulse is important. Of course, every PWS has 
a distribution of vessel sizes, so an optimal compromise must be found: the fluence of the laser must be high enough to destroy the vessels within the exposure time. Thus, the wavelength, pulse duration, and fluence of a laser determine the critical balance between destruction of the target tissue and thermal damage of the surrounding area [6].

To minimize heating of the epidermis, various epidermal cooling techniques have been developed. The challenge is to cool the epidermis selectively while leaving the temperature of the underlying blood vessels unchanged. This epidermal cooling allows higher fluences to be delivered to the PWS and allows thus more photocoagulation of the vessels without thermal damage to the skin. The method that showed most improvement in blanching of PWSs, reduced incidence of adverse effects such as epidermal damage or pigmentary change, and increased patient comfort $[7,8]$ is cryogen spray cooling (CSC) [9], in which spurts of cryogenic fluid are delivered to the skin for tens of milliseconds. In a recent study in Chinese patients, for whom the higher melanin content in the skin poses increased complications, CSC was shown to result in better therapeutic outcome than achieved with laser alone [10]. Cold-air cooling was also shown to be effective [11].

\section{Therapy for port-wine stains}

Various types of lasers have been used to treat PWS over the years: Argon (488 nm, $514 \mathrm{~nm}$ ), Argon-pumped tunable dye laser (APTDL, 577 nm-585 nm), KTP (532 nm), Copper vapor/bromide (578 nm), Krypton (568 nm), and flashlamp-pumped pulsed dye laser (PDL, $585 \mathrm{~nm}$ ). They ranged from continuous wave $(\mathrm{CW})$ lasers (eg, the older Argon laser), to quasi-CW lasers (APTDL, KTP, Copper vapor/bromide, Krypton lasers), to pulsed PDLs $[1,6,8,12]$.

The CW and quasi-CW lasers also have higher incidences of hypertrophic scarring, textural changes, and hyper- or hypo-pigmentation than occur with the PDL [6,8]. Complications of PDL treatment include purpura (bruising) caused by extravasation of red blood cells, postoperative swelling, and postinflammatory hyperpigmentation. These side effects usually resolve within a couple of weeks following treatment.

All laser treatments are performed under some type of anesthesia. Topical anesthesia is usually satisfactory for facial lesions, but sometimes local or regional anesthesia is required. Depending on the lesion size and site, children under 12 years of age may require general anesthesia. Postoperative pain, crusting, swelling, and erythema are alleviated by administering emollients and analgesics [12]. One laser treatment is not usually sufficient to destroy all the vessels that might respond to therapy, so several sessions are always used for best achievable blanching. These treatments are given some weeks apart, so that the epidermis has time to heal. Sixty percent to $80 \%$ improvement in the appearance of a PWS can 
be achieved after 4 to 12 treatments [12], but partial recurrence may occur because of the revitalization of incompletely damaged vessels. Few patients exhibit complete cure, because the threshold for epidermal damage is lower than for permanent blanching of PWS [7]. The site and size of the PWS have been shown to be good predictors of outcome, with small lesions and lesions on the central forehead responding most favorably. The color of the PWS and the age of patient showed conflicting results as predictors [3].

Today, the treatment of choice for PWSs is the PDL at wavelengths from $585 \mathrm{~nm}$ to $600 \mathrm{~nm}$, pulsewidths of 0.4 to 1.5 milliseconds, at repetition rates up to $1 \mathrm{~Hz}$, and fluences of 3 to $10 \mathrm{~J} / \mathrm{cm}^{2}$, with 3 - to $10-\mathrm{mm}$ exposure spots delivered through a fiber-optic handpiece with minimal overlap $[1,3,8]$. Newer laser systems incorporate cryogen cooling in the handpiece, making CSC easy to incorporate in therapy.

The 585-nm/0.45-ms PDL (short-pulse PDL) was developed as a direct result of the photothermolysis theory, based on a calculated $t$ of less than 1 millisecond for vessel diameters of 10 to $50 \mu \mathrm{m}$ [12]. Short-pulse PDL has become the standard mode for PWS treatment but is more effective in pediatric cases, where the vessels are relatively small, than in adult cases, where the vessels grow larger with age. The 560-nm/1.5-ms PDL (long-pulse PDL) was developed to treat deeper and thicker vessels. There are conflicting reports on the effectiveness of the $595-\mathrm{nm} / 1.5-\mathrm{ms}$ system versus the $585-\mathrm{nm} / 0.45-\mathrm{ms}$ system, most likely arising from the different morphology of the particular PWS treated. For deep vessels larger than $1 \mathrm{~mm}$, nearinfrared lasers may be effective as secondary therapy $[3,8]$. One of the most recent advances in PDL offers a tunable wavelength of 585 to $600 \mathrm{~nm}$ at 1.5 milliseconds or a tunable pulse length of 0.5 to 40 milliseconds at a fixed wavelength of $585 \mathrm{~nm}$ or $595 \mathrm{~nm}$ and higher fluences up to $28 \mathrm{~J} / \mathrm{cm}^{2}$.

The KTP laser, at the shorter wavelength of $532 \mathrm{~nm}$ and with pulse durations of 2 to 50 milliseconds, is still used for PWS treatment. Its main advantage is that it causes less purpura than PDL. It has less penetration in tissue, so it is not as effective in treating deeper vessels, and it is absorbed more by melanin and thus is of limited use for patients with darker skin types.

\section{Recent findings on the effect of pulsed dye laser parameters on treatment of port-wine stains}

One recent randomized, prospective study used the latest tunable PDL to compare PWS treatment with two wavelengths $(585 \mathrm{~nm}$ and $595 \mathrm{~nm})$ and two pulse durations ( 0.5 milliseconds and 20 milliseconds) in 15 patients with untreated PWS [13]. Three different laser settings were investigated at a spot size of $7 \mathrm{~mm}: 585 \mathrm{~nm} / 0.5 \mathrm{~ms} / 5.5 \mathrm{~J} / \mathrm{cm}^{2}, 595 \mathrm{~nm} / 0.5 \mathrm{~ms} / 5.5 \mathrm{~J} / \mathrm{cm}^{2}$, and $595 \mathrm{~nm} / 20 \mathrm{~ms} / 13 \mathrm{~J} / \mathrm{cm}^{2}$. Treatments were performed with cold-air cooling. The results showed that the traditional $585-\mathrm{nm} / 0.5-\mathrm{ms}$ system yields a significantly greater clearance of PWSs but also the highest rate of adverse effects. Purple PWSs responded best to this treatment. 
Another study evaluated by histologic observations the thermal damage to ex vivo epidermis samples after irradiation with a 595-nm PDL at various fluences and pulse durations, with and without CSC [14]. The samples were taken from 28 consenting women and had various degrees of pigmentation (Fitzpatrick types I-VI). The laser parameters investigated were incident fluences of $4,6,10,15$, and $20 \mathrm{~J} / \mathrm{cm}^{2}$ and pulse durations of $1.5,10$, and 40 milliseconds. CSC at 100 milliseconds was used. Results showed that under the same incident dosage, longer pulse durations led to decreased thermal injury to the epidermis. CSC allowed use of high incident dosages (15-20 $\mathrm{J} / \mathrm{cm}^{2}$ ) in skin types I through IV without damage to the epidermis. In skin type VI, however, thermal damage could not be prevented even with dosages as low as $4 \mathrm{~J} / \mathrm{cm}^{2}$ with CSC. The article lists the threshold values for irradiation parameters that resulted in thermal injury of each skin type with and without CSC.

Monte Carlo simulations of light-tissue interactions and analytical solutions to the diffusion approximation theory have been used to model PDL treatment of PWS by calculating the temperature distribution and the resulting coagulation pattern within specific blood vessels [15]. Wavelengths in the range of $577 \mathrm{~nm}$ to $587 \mathrm{~nm}$ were suggested as optimal, consistent with the success of PDL in treating PWS. Calculations also predicted that coagulation across vessels was more uniform with $595 \mathrm{~nm}$ than with $585 \mathrm{~nm}$ and that at a depth of $1.2 \mathrm{~mm}$ vessels ranging from 50 to $100 \mu \mathrm{m}$ in diameter would be coagulated from top to bottom, small vessels around $10 \mu \mathrm{m}$ would not be coagulated, and vessels larger than $150 \mu \mathrm{m}$ would be partially coagulated. Thus, this modeling showed that the optimal wavelength depends on the size of the vessel. Consequently, one expects different responses to same treatment from different patients: temperature profiles could be similar, but coagulation depends on the size of the vessels in each person. This variation could explain the widely varying reports in the literature regarding the effectiveness of $585-\mathrm{nm}$ versus $595-\mathrm{nm}$ treatment, which range from a strong preference for one or the other wavelength to seeing no difference between them.

\section{Intense pulsed light source for treatment of port-wine stains}

An alternative to lasers for treatment of PWS has been the intense pulsed light source (IPLS). The IPLS is not a laser, but it works on similar principles. The IPLS is a broadband flashlamp emitting light between 515 and $1200 \mathrm{~nm}$ that is used in conjunction with a series of wavelength-selecting filters. Software enables the operator to choose the treatment parameters, including wavelength and intensity delivered. The IPLS is versatile and can be tailored to various applications, but this versatility makes the establishing of ideal parameters for consistent and reproducible results difficult. Operator skill plays a great role in all laser treatments and an even greater role in IPLS treatment. 
One recent study of 22 patients found that the IPLS can be useful in PWS treatment for Asian patients [16]. Fifteen of the patients had the PWS located over the head and neck region. Treatment consisted of three to eight sessions with 550-nm, 570-nm, and 590-nm cutoff filters in double-pulse or triple-pulse mode in conjunction with chilled gel to protect the epidermis. A longer cutoff filter was used for patients who tanned easily. More than $90 \%$ of patients showed better than $25 \%$ clearing; $40 \%$ of patients showed more than $50 \%$ blanching; and $9 \%$ achieved more than $75 \%$ clearing. No patient exhibited complete blanching. These results were better than the authors' experience with PDL without cooling and showed that IPLS can be effective therapy for PWS in Asian patients. The authors of that report had several years of experience with IPLS before starting this study.

\section{Recent advances in understanding the causes for port-wine stains}

The causes of PWS are not well understood, but in the late 1980s it was proposed that one cause could be the reduction of neural innervation around the vessels [17]. A recent article used a confocal microscope to determine nerve and blood vessel density and mean vessel size in PWSs [18]. A confocal microscope uses the coherent property of lasers (all photons being in phase in both time and space domain) to obtain high-resolution images with resolutions on the order of $\mu \mathrm{m}$. Skin biopsies were taken from untreated PWS, PWS with good response to laser treatment, PWS with poor response to laser treatment, and uninvolved skin of three adult males. Additionally, biopsies of normal skin were obtained from three healthy volunteers of similar age as the patients. Results showed that nerve density was decreased in all evaluated PWS sites and thus could be a factor in lesion pathogenesis. PWS blood vessel size correlated well with response to PDL and might prove to be a useful prognostic indicator of therapeutic outcome.

\section{Future of port-wine stain treatment: noninvasive in vivo imaging or a combination of photodynamic and photothermal treatment?}

The average success rate for complete blanching of PWS is less than $25 \%$ [19]. A major limitation of current laser therapy is that there are no objective criteria to guide treatment plans. The color of the PWS and physician experience are two common subjective criteria. Knowledge of detailed skin parameters around the PWS and of the PWS itself could help optimize treatment. One recent article describes the development of a onedimensional computational model for PWS depth profiling using pulsed photothermal radiometry (PPTR) [19]. PPTR measures the time-resolved changes in the blackbody emission of an object after irradiation with a laser pulse. PPTR has been applied by several groups for depth profiling of PWSs, but it also has some limitations [19]. As discussed earlier, light absorption dissipates as heat; thus, it increases the temperature and changes the blackbody emission spectrum. Light absorbed at the surface changes the 
emission immediately, whereas light absorbed deeper in the skin affects emission only after it diffuses toward the skin's surface. The model used a digitized histologic section of a PWS biopsy as the input skin geometry. Parameters extracted from the model were average epidermal thickness, maximum epidermal temperature rise, depth of PWS upper boundary, and depth of maximum PWS temperature increase. Comparison of actual and reconstructed profiles showed a good match for the four parameters. These results indicate that PPTR is promising for PWS depth profiling.

A drastically different approach to improving outcome is to use PDT in conjunction with standard PDL treatment [20]. (A general discussion of the principles of PDT is given later.) PDT shows potential because of unwanted side effects - red blood cell extravasation and local hemorrhage - that occur during treatment of tumors by this method. One of the main disadvantages of PDT is persistent skin photosensitivity, and second-generation drugs that overcome this photosensitivity by rapid metabolic clearance are under development. One such substance, Benzoporphyrin-derivative monoacid (BPD) is particularly useful for treatment of PWSs because of its strong absorbance at $576 \mathrm{~nm}$ and $690 \mathrm{~nm}$. Sequential yellow and red light treatments can achieve progressively deeper PDT action and be useful for treatment of thick PWSs. PDT uses continuous low irradiance over exposures on the order of minutes, and thus the dose effect is cumulative as exposure time is increased. PDT destroys all vessels containing the drug, independent of their size, unlike PDL-induced coagulation, which spares vessels smaller than $20 \mathrm{um}$. Following subtherapeutic PDT exposure with yellow light, the red blood cells are more susceptible to photothermal damage because of transient changes that include thrombus formation at the vessel wall. PDL irradiation then preferentially heats the pretreated vessels already compromised by PDT. Thus, the combined use of PDT and PDL shows promise in the treatment of PWS. An in vivo study using the chick chorioallantoic membrane model resulted in significantly more severe vascular damage following PDT plus PDL than with either treatment alone [21]. Prospective, comparative, and controlled clinical studies against accepted treatment regimes are required to confirm these results.

\section{Photodynamic therapy}

Theory of photodynamic action and considerations for clinical tissue therapeutics

PDT is often described as a form of light-activated chemotherapy. In PDT, cell death and tissue necrosis result from the action of cytotoxic agents (eg, highly reactive singlet oxygen) that are created through the lightinduced chemical reactions of an administered photosensitizer [22]. For example, in 2003 the United States Food and Drug Administration (FDA) approved the use of PDT with the injectable photosensitizer porfimer 
sodium (Photofrin, Wyeth-Ayerst Lederle, Inc., Pearl River, NY; dihematoporphyrin ether (DHE)) for the selective ablation of precancerous lesions in patients with Barrett's esophagus who do not undergo esophagectomy. Studies supporting the approval had demonstrated that patients receiving PDT had a $20 \%$ chance of developing cancer at 2 years after PDT, whereas the control group (those who did not undergo PDT) had a $50 \%$ chance of developing cancer within that time frame.

Several generations of photosensitizers under investigation, including first-generation mixtures such as porfimer sodium and second- and thirdgeneration purified compounds such as 5-aminolevulinic acid (5-ALA) and meta-tetrahydroxyphenylchlorin (m-THPC). Advances in the development of chemically well-defined photosensitizers have enabled localized targeting of subcellular organelles, including mitochondria, for photodestruction [22]. Photosensitizers may be administered to a patient orally, intravenously, or topically. Given sufficient time for adequate biodistribution, photosensitizers tend to accumulate preferentially in target tissues, such as tumors and dysplastic lesions, relative to normal tissues, thereby enabling selective tissue destruction with light.

In addition to photosensitizer biodistribution, other considerations for successful therapeutic intervention using photodynamic action in tissues include light characteristics (wavelength, energy density, and power density) and the mode of light delivery to tissue. These factors can determine the depth to which light penetrates tissues and the overall efficiency of PDT; the ability to conduct quantitative dosimetry monitoring in vivo, which can enable patient-specific therapeutics; and the rate of photosensitizer clearance, which can help to minimize the risk of posttreatment side effects [23].

\section{Selected applications of photodynamic therapy in otolaryngology}

Several case reports and clinical studies regarding the effectiveness of PDT in otolaryngology employing a variety of photosensitizing agents have appeared in the literature. For example, a case report describing the treatment of infiltrating squamous cell carcinoma in the soft palate used PDT as an alternative to radiotherapy or surgery [24]. In this case, the photosensitizer m-THPC was administered intravenously at $0.15 \mathrm{mg} / \mathrm{kg}$ of body weight 96 hours before treatment. The laser used for PDT (a rhodamine dye laser pumped by a Copper-vapor laser) delivered a light dose of energy (density, $20 \mathrm{~J} / \mathrm{cm}^{2}$; power density, $100 \mathrm{~mW} / \mathrm{cm}^{2}$ ) at a wavelength of $652 \mathrm{~nm}$ and over a spot $3 \mathrm{~cm}$ in diameter. These treatment conditions were thought to be capable of achieving $1-\mathrm{cm}$ tumor necrosis, a depth that was more than sufficient for treating the 2- to 3-mm tumor depth that was revealed on CT. After treatment and recovery, the patient experienced no loss of palatal function and had no recurrence of disease at 16 months after PDT. In this case, the only disadvantage of PDT, as opposed 
to conventional radiotherapy or surgery, was the need, typical of PDT interventions, to avoid direct sunlight for a period of time (in this case, 30 days) following administration of the photosensitizing agent to avoid a potentially serious photosensitive reaction.

Another study evaluated the efficacy of PDT for treatment of recurrent nasopharyngeal carcinoma (NPC) in a clinical trial of 12 patients [25], as an alternative to radiotherapy, surgery, or chemotherapy. The patients studied had recurrent NPC after completing a full course of radiotherapy and had no alternate curative therapy available. PDT treatment consisted of an intravenous injection of hematoporphyrin derivative (HPD) at $5 \mathrm{mg} / \mathrm{kg}$ of body weight followed by laser irradiation 48 to 72 hours later. The light source was a Gold vapor laser at a wavelength of $630 \mathrm{~nm}$ (power, 0.5-1.0 W) that delivered a total energy density of 150 to $200 \mathrm{~J} / \mathrm{cm}^{2}$ during treatment. Light delivery was achieved using an optical fiber with a rounded bulbdiffuser tip. Because the penetration of light into tissue was estimated to be approximately $1 \mathrm{~cm}$, tumors with thicknesses greater than $1 \mathrm{~cm}$ were debulked 2 weeks before PDT. The authors noted four distinct phases of recovery after PDT: (1) an acute, local inflammatory reaction lasting 3 to 4 days; (2) an exudative phase lasting 2 weeks; (3) a crusting phase lasting 3 to 6 months; (4) re-epithelialization to normal-appearing mucosa. At 6 months after PDT all 12 patients showed tumor regression, as confirmed by CT or MRI, and 3 were disease-free at 9 to 12 months follow-up. The authors concluded that even a single treatment of PDT can result in useful palliation or cure for patients with recurrent NPC, with minimal complications (skin hypersensitivity; experienced by two patients).

Although side effects associated with PDT treatment are rare and are largely preventable by avoiding patient exposure to sunlight or bright room light, adverse reactions can occur, and the procedure is not without risk. For example, a case report describes a patient who received PDT for recurrent larynx papillomatosis using the photosensitizer Photosan 3 (DHE) that was administered intravenously at $2.5 \mathrm{mg} / \mathrm{kg}$ of body weight 24 hours before laser irradiation with light from an Argon-dye laser at wavelength $630 \mathrm{~nm}$, administered with a cylindrical light applicator [26]. No complications arose during the procedure, but 3 hours after PDT the patient was inadvertently exposed to neon light and developed serious anaphylaxis, which was treated immediately and successfully in the ICU where the patient had been recovering. This case illustrates the potential seriousness of side effects associated with PDT and the importance of adequate post-PDT patient monitoring and care.

Indeed, large patient-to-patient variations in response to PDT were observed in a controlled study of tissue damage to both healthy mucosa and early squamous cell carcinoma ( $<2 \mathrm{~mm}$ infiltration depth) in the esophagus, bronchi, and mouth using the photosensitizer m-THPC [27]. The study examined 25 patients and found that the optimal conditions for PDT (ie, maximal tumor necrosis with minimal damage to normal tissue) in the 
bronchi and mouth included an intravenously injected dose of $0.15 \mathrm{mg} / \mathrm{kg}$ of body weight of m-THPC followed 4 days later by laser irradiation at wavelength $652 \mathrm{~nm}$ with an Argon-ion PDL with an energy density of 7 to $16 \mathrm{~J} / \mathrm{cm}^{2}$ (power density, $100-150 \mathrm{~mW} / \mathrm{cm}^{2}$ ). In the esophagus, light from an Argon-ion laser at wavelength $514 \mathrm{~nm}$ and at an energy density of 75 to $100 \mathrm{~J} / \mathrm{cm}^{2}$ (power density, $70-100 \mathrm{~mW} / \mathrm{cm}^{2}$ ) was found to be optimal. The second-generation photosensitizer m-THPC, a pure compound, was determined to have several advantages over first-generation porphyrin mixtures such as HPD and porfimer sodium (Photofrin II). These advantages included enhanced phototoxicity (100 times more phototoxic at $652 \mathrm{~nm} ; 10$ times more phototoxic at $514 \mathrm{~nm}$ ) and a shorter duration of skin photosensitivity (one third the time). Of the 33 lesions treated, 28 exhibited no recurrence at 14 months after PDT, giving a recurrence rate similar to that of Photofrin II, approximately $15 \%$. In determining the optimal conditions for PDT, the study found large interindividual variations in response (ranging from no reaction to tissue necrosis) for identical treatment conditions. This variation highlights the need for individualized patient dosimetry and monitoring in PDT. This study and others [23] have suggested the use of photosensitizer spectrofluorometry as a means of quantitatively and noninvasively monitoring photosensitizer concentration in living tissues to guide dosimetry and improve patient response to PDT interventions.

There is much interest in using PDT as an alternative to surgery or radiotherapy for the destruction of high-grade dysplasia and early carcinoma in the esophagus. Recent FDA approval for the first-generation photosensitizer, porfimer sodium, for PDT of precancerous lesions in the esophagus was described earlier. A prospective study of porfimer sodium PDT in 102 patients with advanced and early-stage esophageal cancer found that PDT offered significant improvement of symptoms in patients with advanced disease and was curative in some cases of early disease [28]. In the study, patients received an intravenous injection of porfimer sodium (Photofrin) at $2 \mathrm{mg} / \mathrm{kg}$ of body weight followed 24 to 72 hours later by laser irradiation at wavelength $630 \mathrm{~nm}$. Complications following treatment included photosensitive reactions in the skin (in 5\% of patients) and esophageal stricture (in $8 \%$ of patients). Patient response to PDT was compared with more than 1100 patients who were treated with other methods. For patients with advanced disease, PDT treatment was determined to be as beneficial as other palliative therapies, including esophageal resection, stents, and radiotherapy. For patients with early-stage disease, mean survival after PDT was long term (60.5 months). The study concluded that PDT can offer early-stage patients who are not candidates for esophageal resection the potential for cure.

Another study in the esophagus examined the second-generation photosensitizer 5-ALA for PDT in 27 patients with high-grade dysplasia and early carcinoma [29]. In the study, 5-ALA was administered orally at 
$60 \mathrm{mg} / \mathrm{kg}$ of body weight, and PDT was conducted 4 to 6 hours later using a dye laser operating at wavelength $635 \mathrm{~nm}$. The laser was coupled to an optical fiber with a cylindrical light diffuser $2.0 \mathrm{~cm}$ in length and a light dose of energy (density, $150 \mathrm{~J} / \mathrm{cm}^{2}$; power density, $100 \mathrm{~mW} / \mathrm{cm}^{2}$ ) was employed for PDT. To avoid complications from skin photosensitivity, patients were protected from sunlight and bright room lights for 36 hours after photosensitizer administration. The pilot study found that high-grade dysplasia and superficial carcinoma $(\leq 2 \mathrm{~mm})$ could be completely eradicated by 5-ALA PDT without the serious complications (eg, esophageal strictures) observed in porfimer sodium PDT. This result was attributed to the highly specific accumulation of 5-ALA in dysplastic and malignant mucosa, in contrast to the less selective accumulation of porfimer sodium in esophageal tissues. It was suggested that under the experimental protocol employed in the study, 5-ALA PDT in the esophagus might be limited in effectiveness to mucosal penetration depths of $2 \mathrm{~mm}$ or less; patients with tumor thickness greater than $2 \mathrm{~mm}$ might be treated more successfully by using a different photosensitizer (ie, m-THPC).

\section{Future directions for photodynamic therapy in otolaryngology}

Several promising applications of PDT in otolaryngology have been described. First-generation photosensitizers have been approved by the FDA for use in selected PDT applications. With continued clinical research into the optimal conditions and protocols for using chemically defined second- and third-generation photosensitizers, and with the ability to employ photosensitizer spectrofluorometric methods in vivo to monitor dosimetry quantitatively and noninvasively, the efficiency, selectivity, and specificity of PDT interventions should continue to improve.

For PDT to develop from an experimental therapy to widespread clinical use, its cost effectiveness must also be considered. One prohibitive expense might be the relatively high costs of purchasing and maintaining the advanced laser systems employed to date in research studies. It is probable, however, that these technology costs will decline once laser specifications are well defined and mass production of solid-state laser systems becomes feasible.

In the United States, the costs of 5-ALA PDT and associated endoscopies for esophageal disease have been estimated to be approximately $\$ 6500$ [29], much lower than costs associated with esophageal surgery. For patients with advanced head and neck cancer, a recent study in the United Kingdom compared the cost effectiveness of PDT with the photosensitizer Foscan (Biolitec Pharma, Ltd., Edinburgh, UK) (m-THPC) with palliative chemotherapy, extensive palliative surgery, and no treatment [30]. For each intervention the primary outcome for the study was defined as the incremental cost/life-year saved, relative to no treatment. In comparison with palliative chemotherapy and extensive palliative surgery, Foscan PDT was determined to be the most cost-effective intervention for patients with 
advanced head and neck cancer. The results of these types of analyses, coupled with promising clinical studies such as those described earlier, suggest that PDT might become a viable and cost-effective clinical intervention in otolaryngology.

\section{Summary}

This article has discussed selected applications of laser therapies in otolaryngology. Lasers are the treatment of choice for PWS lesions, but new advances are needed to achieve complete blanching in the majority of cases. Noninvasive in vivo imaging and laser therapy coupled with PDT are two areas showing promise to improve outcome. PDT has the potential to offer new, cost-effective treatment options, and possibly cure, to cancer patients who are not candidates for traditional surgery, chemotherapy, and radiotherapy.

\section{References}

[1] Reinisch L. Laser physics and tissue interactions. Otolaryngol Clin North Am 1996;29(6): 893-912.

[2] Reinisch L. Lasers in otolaryngology. In: Vo-Dinh T, editor. Biomedical photonics handbook. New York (NY): CRC Press; 2003. p. 44-1-44-10.

[3] Suthamjariya K, Anderson RR. Lasers in dermatology. In: Vo-Dinh T, editor. Biomedical photonics handbook. CRC Press; 2003. p. 40-1-40-28.

[4] Stratigos AJ, Dover JS. Overview of lasers and their properties. Dermatol Ther 2000;13: $2-16$.

[5] Anderson RR, Parish JA. Selective photothermolysis: precise microsurgery by selective absorption of pulsed radiation. Science 1983;220:524-7.

[6] Alster TS, Lupton JR. Lasers in dermatology - an overview of types and indications. Am J Clin Dermatol 2000;2(5):291-303.

[7] Kelly KM, Nanda VS, Nelson JS. Treatment of port-wine stain birth marks using the $1.5 \mathrm{msec}$ pulsed dye laser at high fluences in conjunction with cryogen spray cooling. Dermatol Surg 2002;28(4):309-13.

[8] Tanzi EL, Lupton JR, Alster TS. Lasers in dermatology: four decades of progress. J Am Acad Dermatol 2003;49:1-31.

[9] Nelson JS, Milner TE, Anvari B, et al. Dynamic cooling of the epidermis during laser port wine stain therapy. Lasers Surg Med 1994;6S:48.

[10] Chiu CH, Chan HHL, Ho WS, et al. Prospective study of pulsed dye laser in conjunction with cryogen spray cooling for treatment of port wine stains in Chinese patients. Dermatol Surg 2003;29:909-15.

[11] Greve B, Hammes S, Raulin C. The effect of cold air cooling on $585 \mathrm{~nm}$ pulsed dye laser treatment of port wine stain. Dermatol Surg 2001;27:633-6.

[12] Acland KM, Barlow RJ. Lasers for the dermatologist. Br J Dermatol 2000;143:244-55.

[13] Greve B, Raulin C. Prospective study of port wine stain treatment with dye laser: comparison of two wavelengths $(585 \mathrm{~nm}$ vs. $595 \mathrm{~nm})$ and two pulse durations $(0.5$ milliseconds vs. 20 milliseconds). Lasers Surg Med 2004;34:168-73.

[14] Dai T, Pikkul BM, Tunnell JW, et al. Thermal response of human skin epidermis in different skin types to 595-nm irradiation and cryogen spray cooling: an ex-vivo study. In: Bass LS, Kollias N, Malek RS, et al, editors. Lasers in surgery: advanced characterization, therapeutics, and systems XIII. Proceedings of the International Society of Optical Engineering 2003;4949:1-10. 
[15] Shafirstein G, Baumler W, Lapidoth M, et al. A new mathematical approach to the diffusion approximation theory for selective photothermolysis modeling and its implication in laser treatment of port-wine stains. Lasers Surg Med 2004;34:335-47.

[16] Ho WS, Ying SY, Chan PC, et al. Treatment of port wine stains with intense pulsed light: a prospective study. Dermatol Surg 2004;30:887-91.

[17] Smoller BR, Rosen S. Port-wine stains: a disease of altered neural modulation of blood vessels? Arch Dermatol 1986;122:177-9.

[18] Selim MM, Kelly KM, Nelson JS, et al. Confocal microscopy study of nerves and blood vessels in untreated and treated port wine stains: preliminary observations. Dermatol Surg 2004;30:892-7.

[19] Choi B, Majaron B, Nelson JS. Computational model to evaluate port wine stain depth profiling using pulsed photothermal radiometry. J Biom Opt 2004;9(2):299-307.

[20] Kimel S, Svaasand LO, Kelly KM, et al. Synergistic photodynamic and photothermal treatment of port wine stain? Laser Surg Med 2004;34:80-2.

[21] Kelly KM, Kimel S, Smith T, et al. Combined photodynamic and photothermal induced injury enhances damage to in vivo model blood vessels. Laser Surg Med 2004;34:407-13.

[22] Henderson BW, Gollnick SO. Mechanistic principles of photodynamic therapy. In: Vo-Dinh T, editor. Biomedical photonics handbook. CRC Press; 2003. p. 36-1-36-27.

[23] Wilson BC, Weersink RA, Lilge L. Fluorescence in photodynamic therapy dosimetry. In: Mycek M-A, Pogue BW, editors. Handbook of biomedical fluorescence. New York: Marcel Dekker; 2003. p. 529-61.

[24] Poate TWJ, Dilkes MG, Kenyon GS. Use of photodynamic therapy for the treatment of squamous cell carcinoma of the soft palate. Br J Oral Maxillofac Surg 1996;34(1):66-8.

[25] Tong MCF, van Hasselt CA, Woo JKS. Preliminary results of photodynamic therapy for recurrent nasopharyngeal carcinoma. Eur Arch Otorhinolaryngol 1996;253:189-92.

[26] Öfner JG, Schlögl H, Kostron H. Unusual adverse reaction in a patient sensitized with Photosan 3. J Photochem Photobiol B Biol 1996;36:183-4.

[27] Savary J-F, Monnier P, Fontolliet C, et al. Photodynamic therapy for early squamous cell carcinomas of the esophagus, bronchi, and mouth with m-tetra(hydroxyphenyl) chlorine. Arch Otolaryngol Head Neck Surg 1997;123:162-8.

[28] Moghissi K, Dixon K. Photodynamic therapy (PDT) in esophageal cancer: a surgical view of its indication based on 14 years experience. Technol Cancer Res Treat 2003;2(4):319-26.

[29] Gossner L, May A, Sroka R, et al. Photodynamic destruction of high grade dysplasia and early carcinoma of the esophagus after the oral administration of 5-aminolevulinic acid. Cancer 1999;86(10):1921-8.

[30] Hopper C, Niziol C, Sidhu M. The cost effectiveness of Foscan mediated photodynamic therapy (Foscan-PDT) compared with extensive palliative surgery and palliative chemotherapy for patients with advanced head and neck cancer in the UK. Oral Oncol 2004;40: $372-82$. 